\title{
On The Design of Mismatched Filters With An Adjustable Matched Filtering Loss
}

\author{
Çag̃atay Candan \\ Middle East Technical University (METU) \\ Department of Electrical and Electronics Engineering \\ Ankara, Turkey. \\ ccandan@metu.edu.tr
}

\begin{abstract}
We present a method for the design of mismatched filters minimizing the interference from unwanted targets (point target or clutter) under the constraint of matched filtering loss. The method method seeks to find the optimum filter minimizing the interference and having a desired cross-correlation with a given transmitter waveform. The method is applied to get the minimum integrated side-lobe level filters and to optimize the receivers of the pulse diversity systems.
\end{abstract}

Index Terms-Mismatched filter, integrated side-lobe level, pulse diversity, Hadamard codes, complementary codes.

\section{INTRODUCTION}

The optimal detector for the detection of a single target under Gaussian white noise is the matched filter. When the target is observed in the presence of Gaussian clutter and noise, the optimal receiver is the whitened matched filter. With the assumption of slowly time-varying clutter, an accurate estimation of its covariance function and the implementation of the whitened matched can be possible. When multiple targets are present, the signal received is the superposition of returns from each target in addition to clutter and noise. For this case, the received signal can be written as follows:

$$
\mathbf{r}=\sqrt{E_{t}} \mathbf{h}_{\mathbf{k}} b_{k}+\sqrt{E_{t}} \underbrace{\sum_{k^{\prime}=1, k^{\prime} \neq k}^{K} \mathbf{h}_{\mathbf{k}^{\prime}} b_{k^{\prime}}+\mathbf{w}}_{\text {colored noise }}
$$

Here $\mathbf{r}, \mathbf{w}$ and $\mathbf{h}_{\mathbf{k}}$ are $N \times 1$ vectors. The vector $\mathbf{r}$ is the collection of slow-time samples from the range-bin of interest, [1]. The vector $\mathbf{h}_{\mathbf{k}}$ is the slow-time samples of the return signal from the kth target with an unknown Doppler shift and delay. The parameter $b_{k}$ is due to the reflection coefficient of an individual target.

We may interpret $b_{k}$ as a random variable and denote its variance as $\sigma_{b_{k}}^{2}$. If the distribution of $b_{k}$ is taken as independent complex Gaussian variables, as in Swerling-1 case; then the term labeled as colored noise becomes Gaussian distributed with zero mean and covariance matrix $\mathbf{C}_{\mathbf{c n}}=\mathbf{H} \boldsymbol{\Lambda}_{\mathbf{b}} \mathbf{H}^{\mathbf{H}}+$ $N_{0} \mathbf{I}$ where $\mathbf{H}=\left[\mathbf{h}_{\mathbf{1}} \mathbf{h}_{\mathbf{2}} \ldots \mathbf{h}_{\mathbf{k}-\mathbf{1}} \mathbf{h}_{\mathbf{k}+\mathbf{1}} \ldots \mathbf{h}_{\mathbf{K}}\right]$ and $\boldsymbol{\Lambda}_{\mathbf{b}}=$ $\operatorname{diag}\left(\sigma_{b_{1}}^{2}, \sigma_{b_{2}}^{2}, \ldots, \sigma_{b_{k-1}}^{2}, \sigma_{r_{b+1}}^{2}, \ldots, \sigma_{b_{K}}^{2}\right)$.

The optimal receiver for the multiple target scenario with Rayleigh distributed power returns is again the whitened matched filter. It should be noted that to implement the whitened matched filter, the average power from each interfering target, $\sigma_{b_{K}}^{2}$ along with its delay and Doppler shift, $\mathbf{h}_{\mathbf{k}^{\prime}}$, should be known a-priori and these parameters should not change during the estimation and coherent processing interval. Even though, such an operation can be feasible in some scenarios; it is a indeed challenging to implement for many targets of interest, [2].

The optimal receiver described for the multi-target scenario can also be labeled as a mismatched filter. The mismatched filters are designed to improve the detection statistics of the target of interest by sacrificing some of the coherent combination gain (matched filter gain). In other words, the mismatched receivers reduce the effect of interference at a cost of losing perfect correlation with the incoming signal.

Since the realization of the optimal mismatched filters is difficult, the sub-optimal filters are designed to reduce the effect of interfering targets known to reside in a pre-defined region in the ambiguity plane. Such applications may arise especially in tracking systems. The goal of mismatch filter design for these applications is to find a filter which has the smallest possible cross-ambiguity surface volume over a predefined region, while sustaining an acceptable correlation with the signal of interest.

In [3], the mismatched filters are constrained to have a correlation coefficient of $\rho$ with the incoming signal and designed to have a minimum possible total energy over a desired region in the ambiguity plane. Here we revisit the problem of Stutt as in [3] and present a novel method for its solution and extend the discussion to mismatch filter design for pulse diversity systems.

In [5] a set of codes and mismatched filters have been designed to have good auto-correlation and cross-correlation values. In [6], [7], the peak side lobe level of the codes has been minimized through iterative algorithms. The minimization of the integrated side lobe level is appropriate when the scatters causing interference have homogeneous reflection powers. The peak side lobe level minimization can be more suitable when interferes are discrete in nature and have varying powers which can be the case for high resolution systems. In [8], [9], the same problem has been examined in the context of SIR (signal-to-interference ratio) maximization.

In this paper, we present a study on mismatched filter 
design following the formalism of Stoica, [8], [9]. In the next section, we illustrate the design procedure for the case where Doppler frequency shift between interfering targets and target of interest is zero. In the following section, we modify the procedure to include the effect of Doppler frequency shift and extend the earlier results to the pulse diversity systems. In this paper, we use the pulse diversity term to refer to the change of pulse shape (code) at every pulse repetition interval (PRI) of coherent processing time (CPI) as in [4].

\section{Mismatched Filter Design (Zero Doppler CASE)}

We start with the preliminary definitions. The $k$ th crosscorrelation lag of vectors $\mathbf{s}$ and $\mathbf{w}$ is denoted as $r_{w s}(k)=$ $\sum w[n] s^{*}[n-k]$. When vectors $\mathbf{s}$ and $\mathbf{w}$ are of finite dimensions, $r_{w s}(k)$ can be expressed as the inner product of two finite dimensional vectors, $r_{w s}(k)=\left(\mathbf{J}_{\mathbf{k}} \mathbf{s}\right)^{H} \mathbf{w}$. Here $\mathbf{J}_{\mathbf{k}}$ is the shift matrix.

The matrix given below shows a 5 dimensional $\mathbf{J}_{2}$ matrix shifting the input by 2 units, that is $\mathbf{J}_{\mathbf{2}}[a b c d e]^{T}=$ $\left[\begin{array}{lllll}0 & 0 & a & b & c\end{array}\right]^{T}$ :

$$
\mathbf{J}_{\mathbf{2}}=\left[\begin{array}{lllll}
0 & 0 & 0 & 0 & 0 \\
0 & 0 & 0 & 0 & 0 \\
1 & 0 & 0 & 0 & 0 \\
0 & 1 & 0 & 0 & 0 \\
0 & 0 & 1 & 0 & 0
\end{array}\right]
$$

As an example, the second auto-correlation lag of $s=$ $\left[\begin{array}{lllll}a & b & c & d & e\end{array}\right]^{T}$ can be written as $\left(\mathbf{J}_{\mathbf{2}} \mathbf{s}\right)^{\mathbf{H}} \mathbf{s}=\mathbf{s}^{\mathbf{H}} \mathbf{J}_{\mathbf{2}}^{\mathbf{H}} \mathbf{s}=c a^{*}+$ $d b^{*}+e c^{*}$.

Following [9], we can write the total interference of an unwanted targets at $k_{1}, k_{2}, \ldots, k_{L}$ range cells away from the target of interest as follows:

$$
\begin{aligned}
\mathrm{I}_{\text {total }}=\sum_{i=1}^{L} \underbrace{\left|r_{w s}\left(k_{i}\right)\right|^{2}}_{\mathrm{I}_{\mathrm{i}}} & =\sum_{i=1}^{L}\left|\left(\mathbf{J}_{\mathbf{k}_{\mathbf{i}}} \mathbf{s}\right)^{\mathbf{H}} \mathbf{w}\right|^{2} \\
& =\sum_{i=1}^{L} \mathbf{w}^{\mathbf{H}}\left(\mathbf{J}_{\mathbf{k}_{\mathbf{i}}} \mathbf{s}\right)\left(\mathbf{J}_{\mathbf{k}_{\mathbf{i}}} \mathbf{s}\right)^{\mathbf{H}} \mathbf{w} \\
& =\mathbf{w}^{\mathbf{H}} \mathbf{R} \mathbf{w}
\end{aligned}
$$

where

$$
\mathbf{R}=\sum_{i=1}^{L}\left(\mathbf{J}_{\mathbf{k}_{\mathbf{i}}} \mathbf{s}\right)\left(\mathbf{J}_{\mathbf{k}_{\mathbf{i}}} \mathbf{s}\right)^{\mathbf{H}}=\sum_{i=1}^{L} \mathbf{J}_{\mathbf{k}_{\mathbf{i}}} \mathbf{s} \mathbf{s}^{\mathbf{H}} \mathbf{J}_{\mathbf{k}_{\mathbf{i}}} \mathbf{H}
$$

Here $\mathbf{w}$ is the filter used at the receiver, and when $\mathbf{w}=\mathbf{s}$, we have the matched filter. As noted before, in this section we assume that all targets (wanted and unwanted) have the same Doppler frequency shift, this unrealistic assumption for radar systems is removed in the next section.

The mismatched filter design problem can be posed as the minimization of the interference level given in (1) under the constraints of $\mathbf{w}^{\mathbf{H}} \mathbf{w}=1$ and $\left|\mathbf{w}^{\mathbf{H}} \mathbf{s}\right|^{2}=\rho^{2}(|\rho| \leq 1)$. Here it is assumed that $\mathbf{s}$ is normalized such that $\|\mathbf{s}\|^{2}=\mathbf{s}^{\mathbf{H}} \mathbf{S}=1$, therefore $|\rho|$ is the correlation coefficient or the cosine of the angle between vectors $\mathbf{s}$ and $\mathbf{w}$ :

$$
\min _{\mathbf{w}} \mathbf{w}^{\mathbf{H}} \mathbf{R} \mathbf{w} \quad \text { s.t. } \quad \begin{array}{ll}
\left|\mathbf{w}^{\mathbf{H}} \mathbf{S}\right|^{2}=\rho^{2} \quad \text { where } \quad|\rho| \leq 1 \\
\mathbf{w}^{\mathbf{H}} \mathbf{w}=1
\end{array}
$$

A solution for the problem can be produced as follows: Let $\mathbf{w}$ be the solution of the problem, then $\mathbf{w}$ can be written as follows:

$$
\mathbf{w}=\rho \mathbf{s}+x_{1} \mathbf{u}_{\mathbf{1}}+x_{2} \mathbf{u}_{\mathbf{2}}+\ldots+x_{N-1} \mathbf{u}_{\mathbf{N}-\mathbf{1}}
$$

The vectors $\left\{\mathbf{s}, \mathbf{u}_{\mathbf{1}}, \ldots, \mathbf{u}_{\mathbf{N}-\mathbf{1}}\right\}$ form an orthonormal basis for the $\mathrm{N}$ dimensional vector space. Such a basis can be easily generated using Gram-Schmidt procedure.

Note that when $\mathbf{w}$ is decomposed as in (4) then the constraint of $\left|\mathbf{w}^{\mathbf{H}} \mathbf{s}\right|^{2}=\rho^{2}$ is immediately satisfied. The second constraint $\mathbf{w}^{\mathbf{H}} \mathbf{W}=1$ under this decomposition reduces to $\mathbf{w}^{\mathbf{H}} \mathbf{w}=\rho^{2}+\mathbf{x}^{\mathbf{H}} \mathbf{x}=1$. Here $\mathbf{x}$ is $(N-1)$ dimensional column vector composed of $\left\{x_{1}, x_{2}, \ldots, x_{N-1}\right\}$ coefficients given in (4).

The optimization problem reduces to the following problem:

$$
\min _{\mathbf{x}}(\rho \mathbf{s}+\mathbf{U} \mathbf{x})^{\mathbf{H}} \mathbf{R}(\rho \mathbf{s}+\mathbf{U} \mathbf{x}) \quad \text { s.t. } \quad \mathbf{x}^{\mathbf{H}} \mathbf{x}=1-\rho^{2}
$$

Once the optimal $\mathbf{x}$ can be found, the optimal mismatched filter can be written using (4).

The constrained optimization problem can also be expressed as an unconstrained optimization problem using Lagrange multipliers:

$\mathrm{J}(\mathbf{x}, \gamma)=(\rho \mathbf{s}+\mathbf{U} \mathbf{x})^{\mathbf{H}} \mathbf{R}(\rho \mathbf{s}+\mathbf{U} \mathbf{x})+\gamma\left(\mathbf{x}^{\mathbf{H}} \mathbf{x}-\left(1-\rho^{2}\right)\right)$

Here $\gamma$ is the Lagrange multiplier. When gradient of $\mathrm{J}(\mathbf{x}, \gamma)$ is calculated (with respect to $\mathbf{x}$ ) and equated to zero, we get the following condition for the optimal weights:

$$
\left(\mathbf{U}^{\mathbf{H}} \mathbf{R U}+\gamma \mathbf{I}\right) \mathbf{x}=\rho \mathbf{U}^{\mathbf{H}} \mathbf{R} \mathbf{s}
$$

The equation (7) has different solutions for different values of Lagrange multiplier $\gamma$. The $\gamma$ parameter should be selected such that the constraint of $\mathbf{x}^{\mathbf{H}} \mathbf{x}=1-\rho^{2}$ is satisfied and at the same time the overall cost is minimized.

To facilitate the calculation of $\gamma$, we decompose the matrix $\mathbf{U}^{\mathbf{H}} \mathbf{R U}$ into its eigenvalues and eigenvectors, that is $\mathbf{U}^{\mathbf{H}} \mathbf{R U}=\mathbf{V} \mathbf{\Lambda} \mathbf{V}^{\mathbf{H}}$. Due to the symmetry of $\mathbf{U}^{\mathbf{H}} \mathbf{R U}$, the matrix $\mathbf{V}$ is unitary and $\boldsymbol{\Lambda}$ is a diagonal matrix with real entries. Then equation (7) can be written as follows:

$$
\mathbf{V}(\boldsymbol{\Lambda}+\gamma \mathbf{I}) \underbrace{\mathbf{V}^{\mathbf{H}} \mathbf{x}}_{\widehat{\mathbf{x}}}=\rho \mathbf{U}^{\mathbf{H}} \mathbf{R s}
$$

Then $\widehat{\mathbf{x}}$ is equal to

$$
(\boldsymbol{\Lambda}+\gamma \mathbf{I}) \widehat{\mathbf{x}}=\rho \underbrace{\mathbf{V}^{\mathbf{H}} \mathbf{U}^{\mathbf{H}} \mathbf{R} \mathbf{s}}_{\mathbf{b}}
$$

and

$$
\widehat{\mathbf{x}}=\rho(\boldsymbol{\Lambda}+\gamma \mathbf{I})^{-1} \mathbf{b}
$$

Note that right hand side of (10) except the $\gamma$ parameter can be calculated from known data. To calculate the $\gamma$ parameter we need to make use of the constraint $\mathbf{x}^{\mathbf{H}} \mathbf{x}=1-\rho^{2}$. 
Since $\mathbf{V}$ matrix is unitary, $\mathbf{x}^{\mathbf{H}} \mathbf{x}=\widehat{\mathbf{x}}^{\mathbf{H}} \widehat{\mathbf{x}}$ and therefore we can immediately use (10) for the calculation of $\gamma$ :

$$
\widehat{\mathbf{x}}^{\mathbf{H}_{\widehat{\mathbf{x}}}}=\sum_{k=1}^{N-1} \frac{\rho^{2}\left|b_{k}\right|^{2}}{\left(\lambda_{k}+\gamma\right)^{2}}=1-\rho^{2}=\mathbf{x}^{\mathbf{H}} \mathbf{x}
$$

The last equation can be written as in a bit more tidy form as follows:

$$
\sum_{k=1}^{N-1} \frac{\left|b_{k}\right|^{2}}{\left(\lambda_{k}+\gamma\right)^{2}}=\frac{1-\rho^{2}}{\rho^{2}}
$$

The values for $\gamma$ satisfying the equality of LHS and RHS of (12) establishes the second constraint.

Among the gamma values, the one minimizes the cost function should be selected to achieve the goal of minimizing the equation (6). Once the optimal $\gamma$ value is found, the mismatched filter can be written as $\mathbf{w}=\rho \mathbf{s}+\mathbf{V} \widehat{\mathbf{x}}=$ $\rho \mathbf{s}+\rho \mathbf{V}(\boldsymbol{\Lambda}+\gamma \mathbf{I})^{-1} \mathbf{b}$.

\section{Mismatched Filter Design (Non-Zero Doppler CASE)}

In this section, we examine the effect of Doppler shift or equivalently radial velocity difference between wanted and unwanted targets. We examine the cases of single pulse, group of identical pulses and, group of diverse pulses.

Effect on a Single Pulse: The change of the signal phase due to Doppler frequency shift for the duration of radar waveform is $2 \pi v_{r} T_{p} / \lambda$. Here $v_{r}$ is the radial velocity of the target, $T_{p}$ is the pulse duration and $\lambda$ is the wavelength of the radar system. In many scenarios, $T_{p}$ is on the order of micro seconds and $\lambda$ is on the order of centimeters. Hence unless $v_{r}$ is extraordinarily large, i.e. on the order of $10^{4} \mathrm{~m} / \mathrm{s}$, the phase change over the pulse duration is insignificant for pulse-Doppler radar systems. In these applications, the effect of Doppler frequency shift on the individual transmissions can be neglected.

Effect on a Group of Identical Pulses: In a conventional pulse-Doppler radar system, a pulse is repeatedly transmitted and the target is detected by coherently combining these receptions. Assuming that $N$ pulses are transmitted during coherent processing interval, then the interference from a target at $k_{i}$ cells away from the range cell of interest and having a phase increment of $\phi$ per PRI interval, i.e. $\phi_{i}=2 \pi v_{r}(\mathrm{PRI}) / \lambda$, can be written as

$$
\begin{aligned}
\mathrm{I}_{\mathrm{i}}=\left|\sum_{p=0}^{N-1} e^{j p \phi_{i}}\left(\mathbf{J}_{\mathbf{k}_{\mathbf{i}}} \mathbf{s}\right) \mathbf{H}_{\mathbf{w}}\right|^{2} & =\left|\left(\mathbf{J}_{\mathbf{k}_{\mathbf{i}}} \mathbf{s}\right)^{\mathbf{H}} \mathbf{w}\right|^{2}\left|\sum_{p=0}^{N-1} e^{j p \phi_{i}}\right|^{2} \\
& =\left|\left(\mathbf{J}_{\mathbf{k}_{\mathbf{i}}} \mathbf{s}\right)^{\mathbf{H}} \mathbf{w}\right|^{2}\left|\frac{\sin \left(N \phi_{i} / 2\right)}{\sin \left(\phi_{i} / 2\right)}\right|^{2}
\end{aligned}
$$

When there are $L$ interfering targets, the total interference can be written as $\mathrm{I}_{\text {total }}=\mathrm{w}^{\mathbf{H}} \mathbf{R w}$ (similar to (1)) where $\mathbf{R}$ is

$$
\mathbf{R}=\sum_{i=1}^{L}\left(\mathbf{J}_{\mathbf{k}_{\mathbf{i}}} \mathbf{s}\right)\left(\mathbf{J}_{\mathbf{k}_{\mathbf{i}}} \mathbf{s}\right) \mathbf{H}\left|\frac{\sin \left(N \phi_{i} / 2\right)}{\sin \left(\phi_{i} / 2\right)}\right|^{2} .
$$

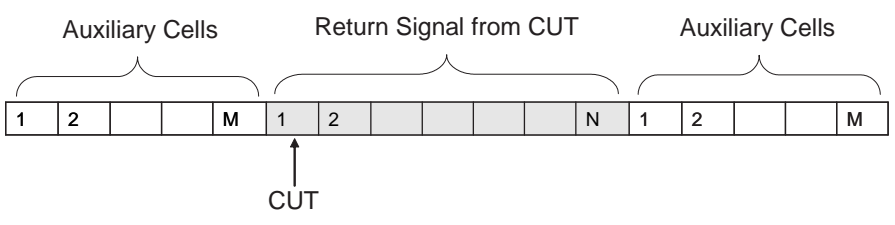

Fig. 1. Cells used for mismatched filtering.

Effect on a Group of Diverse Pulses: A system with pulse diversity is capable of using $N$ different pulses during coherent processing interval. We denote the $k$ th pulse in the train of $N$ pulses with $\mathbf{s}_{\mathbf{k}}$. The receiver processes the individual returns from a range cell with $\mathbf{w}_{k}$ and coherently adds them.

The interference from a target at $k_{i}$ cells away from the range cell of interest and having a phase increment $\phi$ per PRI interval is given in equation (15) (which is on the top of the next page).

When there are $L$ interfering targets, the total interference can be written as $\mathrm{I}_{\text {total }}=\mathrm{w}^{\mathrm{H}} \mathbf{R} \mathbf{w}$ where $\mathbf{R}$ matrix for this case is

$$
\mathbf{R}=\sum_{i=1}^{L} \overline{\mathbf{v}}_{\mathbf{i}} \overline{\mathbf{v}}_{\mathbf{i}} \mathbf{H}
$$

where $\overline{\mathbf{v}}_{\mathbf{i}}$ is given in (15).

It can be observed that by substituting $\mathbf{R}$ matrix from (14) or (16) into the mismatched filter design procedure described for the zero-Doppler shift, we get the procedure for non-zero Doppler shift.

\section{NUMERICAL COMPARISONS}

In the first experiment, the integrated sidelobe level (ISL) of Barker code of length 13 is optimized using mismatched filters. In ISL optimization the Doppler shift of cell under test (CUT) and interfering cells are taken as zero. The definition of ISL is given as follows:

$$
\text { ISL }=\sum_{k=-(N+M-1), k \neq 0}^{N+M-1}\left|r_{w s}(k)\right|^{2}
$$

Here $N$ is the length of the code which is 13 for Barker sequence and $M$ is the number of auxiliary cells which appear before and after the return signal from the CUT as shown in Figure 1 . The auxiliary cells are used to reject the interference from neighboring targets. In ISL calculation, every cell shown in Figure 1, except CUT, is implicitly assumed to have an equal power interfering target.

When noise in the system is negligible in comparison to the clutter, i.e. the variance of noise in (1) is small in comparison to the power return from unwanted targets; then the signal to clutter ratio $(\mathrm{SCR})$ is the factor determining the detection

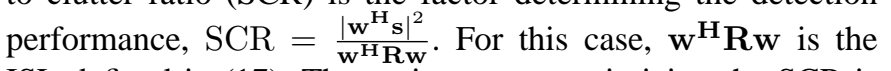
ISL defined in (17). The optimum $w$ maximizing the SCR is $\mathbf{R}^{-1} \mathbf{s}$ and the achievable maximum SCR is $\mathbf{s}^{\mathbf{H}} \mathbf{R}^{-1} \mathbf{s}$, [8], [9].

Figure 2 shows the ISL improvement factor and matched filtering loss curve for the procedure defined in this paper. 
$\mathrm{I}_{\mathrm{i}}=\left|\sum_{p=0}^{N-1} e^{j p \phi_{i}}\left(\mathbf{J}_{\mathbf{k}_{\mathbf{i}}} \mathbf{s}_{\mathbf{p}}\right)^{\mathbf{H}} \mathbf{w}_{\mathbf{p}}\right|^{2}=|\underbrace{\left[\left(\mathbf{J}_{\mathbf{k}_{\mathbf{i}}} \mathbf{s}_{\mathbf{0}}\right)^{H}, e^{j p \phi_{i}}\left(\mathbf{J}_{\mathbf{k}_{\mathbf{i}}} \mathbf{s}_{\mathbf{1}}\right)^{H}, \ldots, e^{j(N-1) p \phi_{i}}\left(\mathbf{J}_{\mathbf{k}_{\mathbf{i}}} \mathbf{S}_{\mathbf{N}-\mathbf{1}}\right)^{H}\right]}_{\overline{\mathbf{v}} \mathbf{H}} \underbrace{\left[\begin{array}{c}\mathbf{w}_{\mathbf{0}} \\ \mathbf{w}_{\mathbf{1}} \\ \ldots \\ \mathbf{w}_{\mathbf{N}-\mathbf{1}}\end{array}\right]}_{\overline{\mathbf{w}}}|^{2}=\overline{\mathbf{w}}^{\mathbf{H}} \overline{\mathbf{v}}_{\mathbf{i}} \overline{\mathbf{v}}_{\mathbf{i}} \mathbf{H}_{\overline{\mathbf{w}}}(15)$

(The ISL improvement factor is defined as the ratio of the ISL level achieved by matched filtering and the ISL level achieved by mismatched filtering. Matched filtering loss (MF loss) is the loss due to the mismatch of $\mathbf{w}$ and $\mathbf{s}$ and it is expressed in decibels as $20 \log _{10}(|\rho|)$.) In the same figure, the ISL improvement of maximum SCR filter [8] is also shown. The SCR improvement over matched filtering is the ISL improvement factor minus matched filtering loss (in decibels). As can be noted from Figure 2, ISL improvement factor remains at negative values (no improvement) at insufficient MF loss values. ISL improvement suddenly changes to the maximum position at an incremental amount of additional MF loss indicating the non-linearity of the optimization procedure. We would like to mention that maximum SCR filter of [8] is a unique filter defined by $\mathbf{R}^{-1} \mathbf{s}$ whose MF loss amount can not be controlled. The filters designed through the proposed method has adjustable MF loss amount and these filters are identical to the maximum SCR filter for a particular value of MF loss value as seen from Figure 2.

Figure 3 shows the cross-correlation of mismatched filter and Barker- 13 code at three different MF Loss values. The selected MF Loss values are shown with circles in Figure 2. As can be noted from Figure 3, insufficient MF loss amount can be more harmful to ISL level than beneficial.

In the second experiment, the effect of Doppler frequency difference in the design of mismatched filters are examined. The radar waveform is composed of the columns of Hadamard matrix. It is known that the columns of Hadamard matrix form an orthogonal set and have complementary auto-correlation, [4, p.269]. In this experiment we use $16 \times 16$ Hadamard matrix and design a mismatched filter of length 26 for each column of Hadamard matrix.

Figure 4 shows the magnitude of the ambiguity function for $16 \times 16$ Hadamard code. The ambiguity function used in the experiment is defined as follows:

$$
A_{s w}(k, \phi)=\sum_{p=1}^{N_{p}}\left(e^{j \phi p} \sum_{k=1}^{N} s_{k}[n] w^{*}[n-k]\right)
$$

Here the phase progression during the pulse duration is assumed to be negligible as discussed before. $N_{p}$ is the number of pulses in CPI and $N$ is the length of the code. For $16 \times 16$ Hadamard system, both parameters are equal to 16 . When we take $s[n]=w[n]$ in (18), the resulting expression is called the self-ambiguity function and the cross-ambiguity function otherwise.
From Figure $4^{1}$ it can be observed that the self-ambiguity function contains localized peaks. Such a peaky characteristic is a set back on the applicability of the pulse diversity systems. In Figure 5, a mismatched filter has been designed to suppress the components of the 4th lag in the self-ambiguity figure. The design has $1.51 \mathrm{~dB}$ matched filtering loss, but suppresses the interference caused by the 4th lag components by $52 \mathrm{~dB}$. In Figure 6, another mismatched filter has been designed to suppress components of both 4th and 8th lags. This design has $2.51 \mathrm{~dB}$ matched filtering loss, but suppresses the overall contribution from both 4th and 8th lags by $90 \mathrm{~dB}$.

\section{CONCLUSION}

We have presented a method for the design of mismatched filters. The method allows to set a desired value for the matched filtering loss and minimizes the interference by unwanted targets (or clutter) under this constraint. The proposed method provides a mechanism to explicitly control the loss due to mismatch of transmit waveform and receiver filter. The method can be useful for the systems where the matched filtering loss along with the signal to clutter ratio is of concern.

\section{ACKNOWLEDGMENT}

Author acknowledges the support of TUBITAK for this work under grant no. 106E187.

\section{REFERENCES}

[1] M. A. Richards, Fundamentals of Radar Signal Processing. New York: McGraw-Hill, 2005.

[2] H. L. V. Trees, Detection, Estimation and Modulation Theory, part 3. John Wiley - Sons, 1971.

[3] C. Stutt and L. Spafford, "A 'best' mismatched filter response for radar clutter discrimination," IEEE Trans. Information Theory, vol. 14, pp. 280287, Mar 1968.

[4] N. Levanon and E. Mozeson, Radar Signals. Hoboken, NJ: WileyInterscience and IEEE Press, 2004.

[5] K. Griep, J. Ritcey, and J. Burlingame, "Poly-phase codes and optimal filters for multiple user ranging," IEEE Trans. Aerospace and Electronic Systems, vol. 31, pp. 752-767, Apr 1995.

[6] A. Zejak, E. Zentner, and P. Rapajic, "Doppler optimised mismatched filters," Electronics Letters, vol. 27, pp. 558-560, March 1991.

[7] S. Zoraster, "Minimum peak range sidelobe filters for binary phase-coded waveforms," IEEE Trans. Aerospace and Electronic Systems, vol. AES16, pp. 112-115, Jan. 1980.

[8] P. Stoica, J. Li, and M. Xue, "Transmit codes and receive filters for radar," IEEE Signal Processing Magazine, vol. 25, pp. 94-109, November 2008.

[9] P. Stoica, J. Li, and M. Xue, "On binary probing signals and instrumental variables receivers for radar," IEEE Trans. Information Theory, vol. 54, pp. 3820-3825, Aug. 2008.

${ }^{1}$ Figures of the paper in color are available at http://ieeexplore.ieee.org. MATLAB .fig files for Figures 4, 5 and 6 (for 3-dimensional viewing) are available at http://www.eee.metu.edu.tr/ $\sim$ ccandan/pub_dir/figures/radar2010/ and are individually available by clicking on the links given in the captions. 


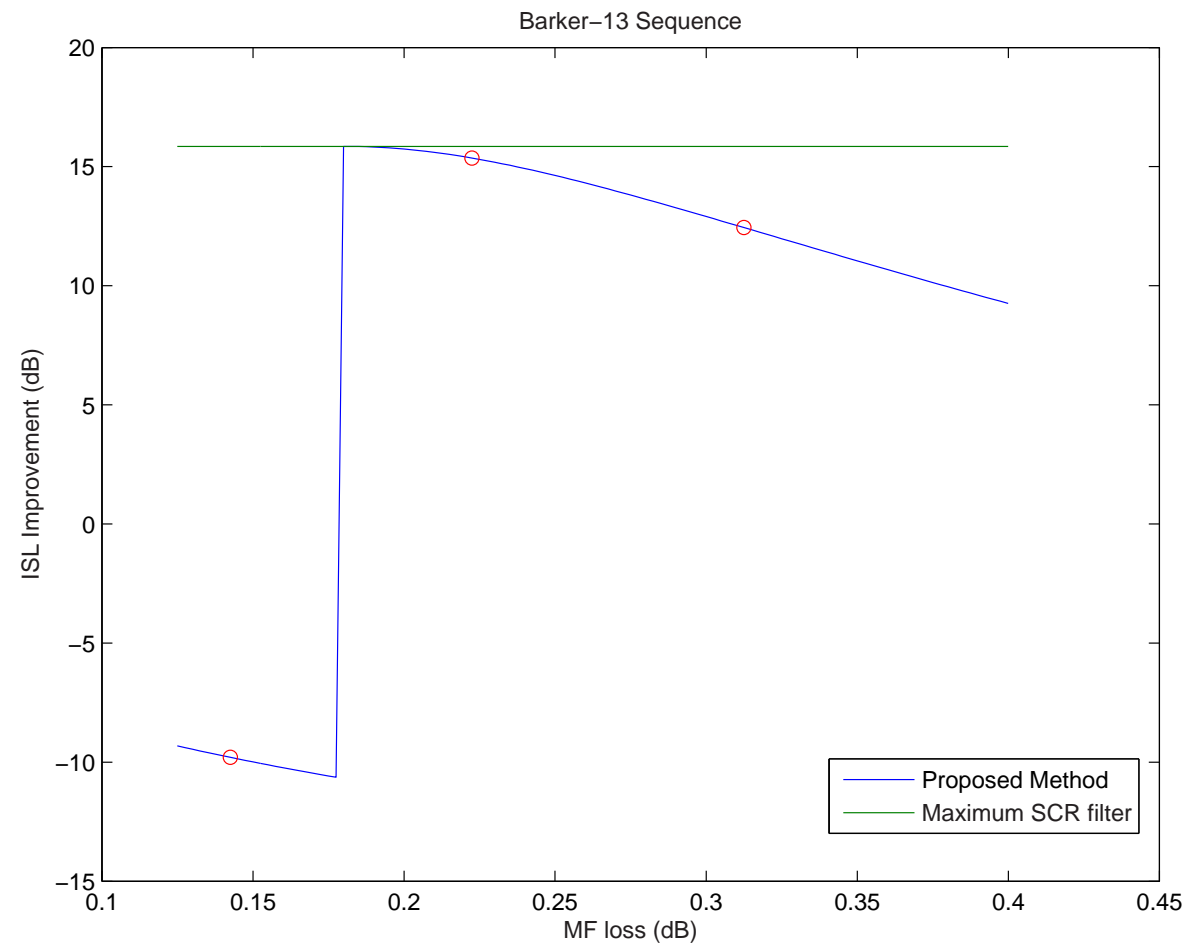

Fig. 2. ISL Improvement factor at different levels of tolerable matched filter loss for Barker waveform of length 13. Circles denote the designs selected for Figure 3.

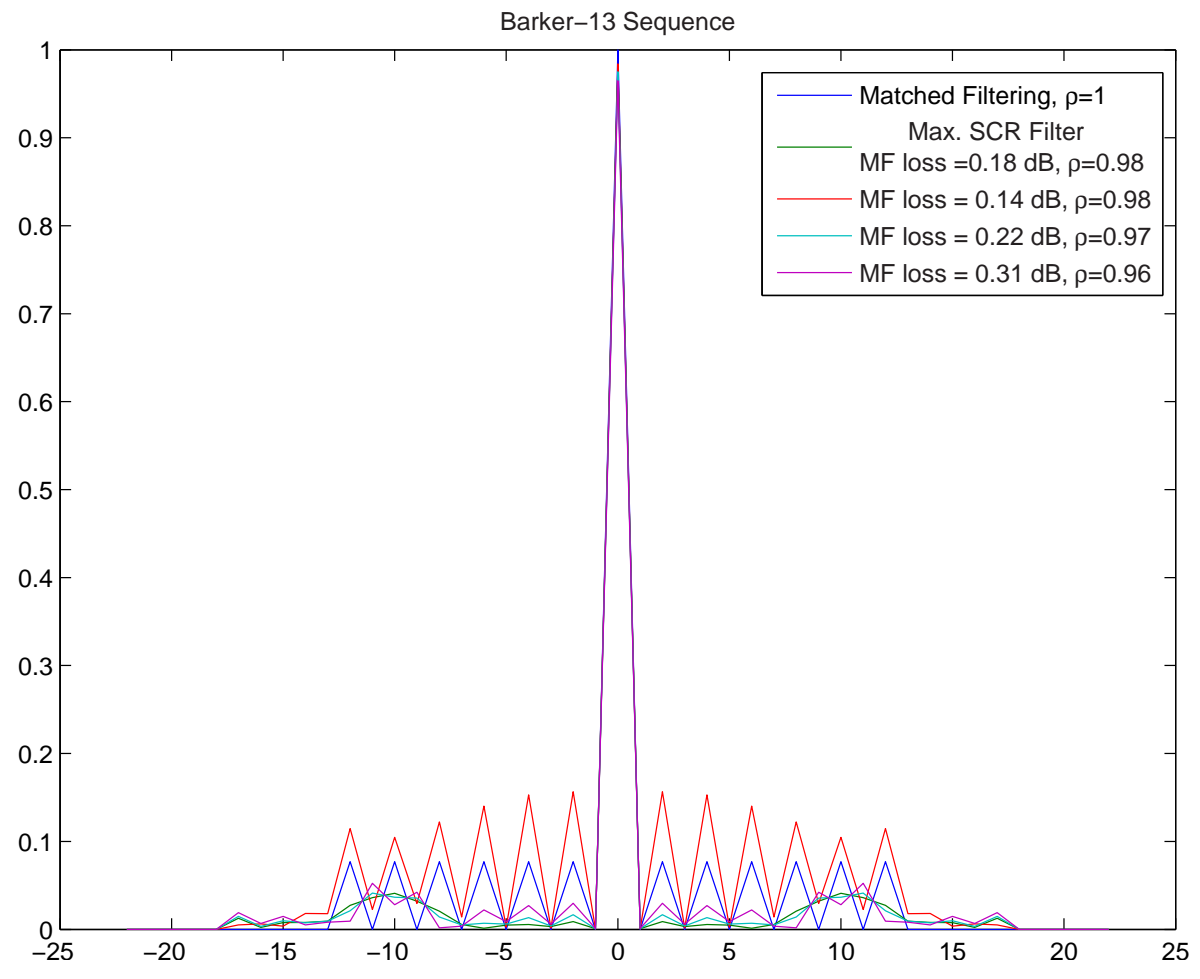

Fig. 3. Cross-correlation of mismatched filter of length 23 and Barker waveform of length 13. 


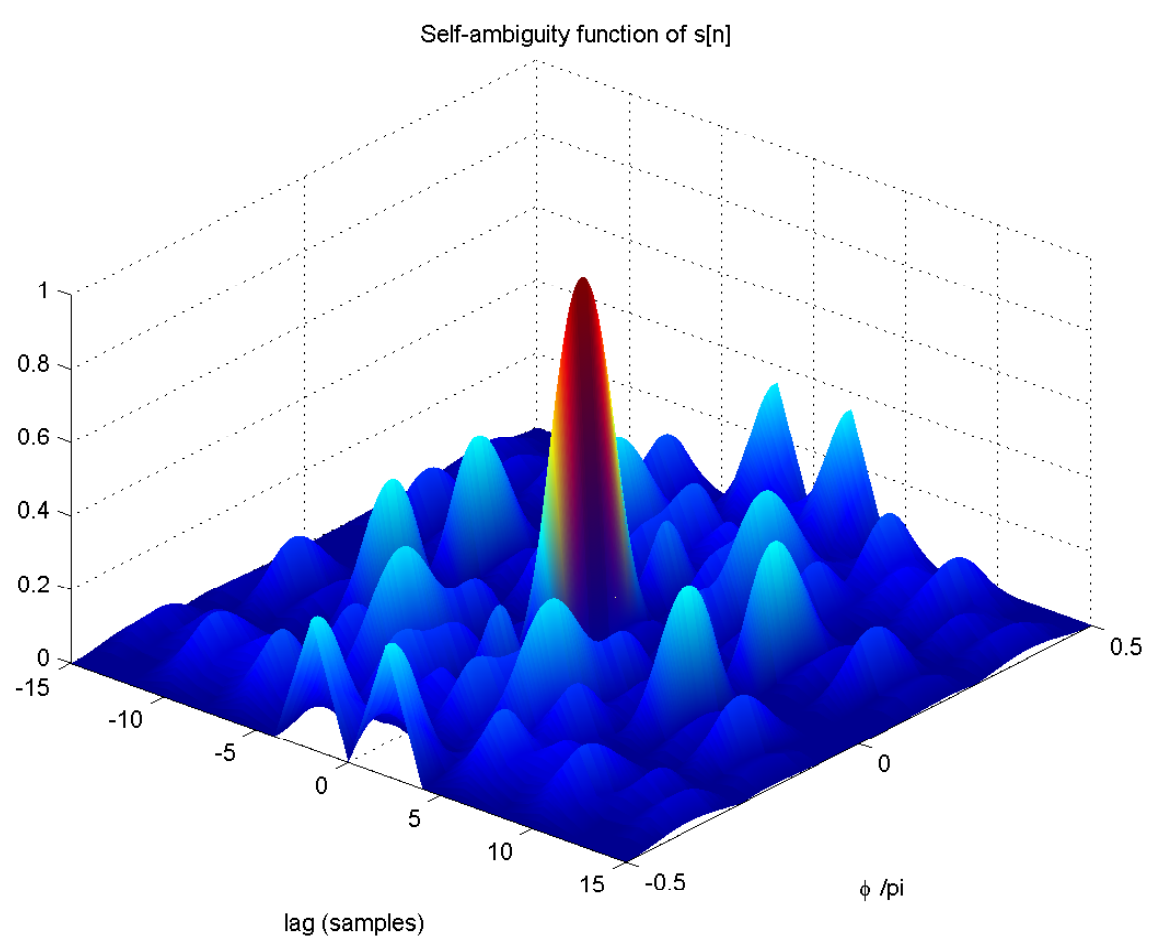

Fig. 4. Self-ambiguity function of $16 \times 16$ Hadamard code. [URL link for figure].

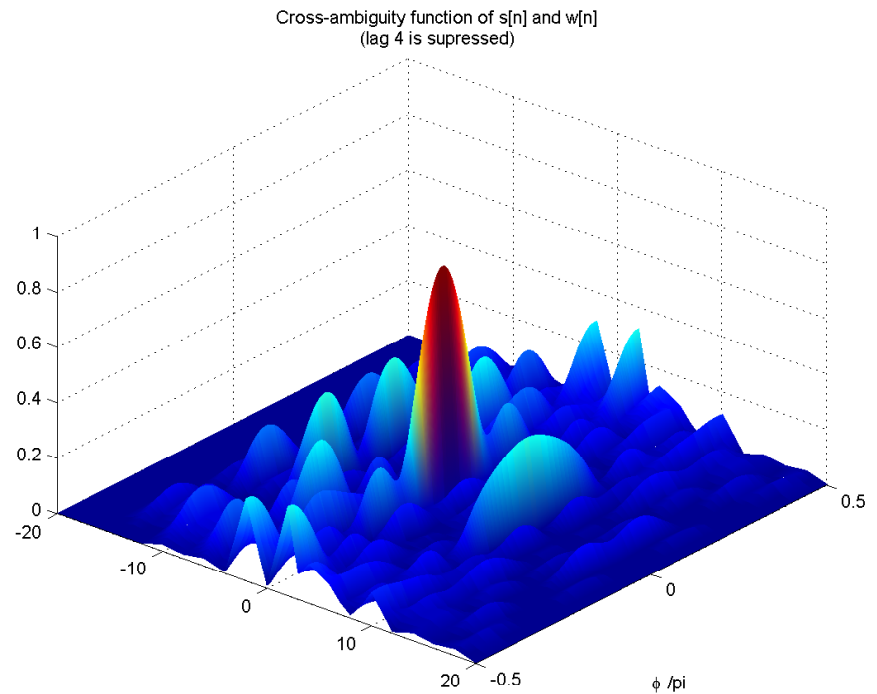

lag (samples)

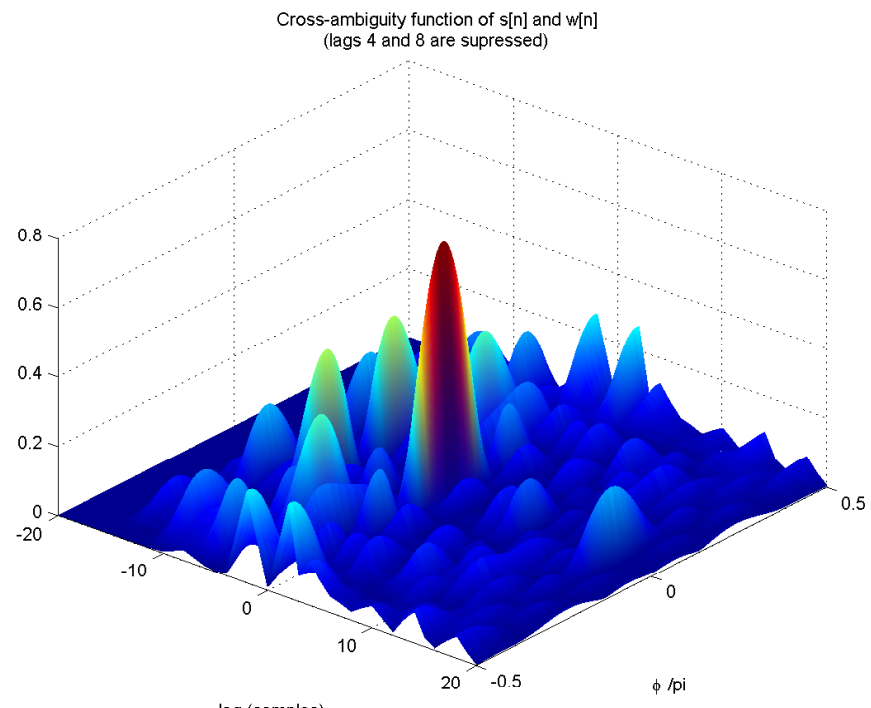

lag (samples)
Fig. 5. Cross-ambiguity function of $16 \times 16$ Hadamard code with the mismatched filter designed to suppress the contributions from the 4th lag, [URL link for figure].
Fig. 6. Cross-ambiguity function of $16 \times 16$ Hadamard code with the mismatched filter designed to suppress the contributions from 4th and 8th lags. [URL link for figure]. 